\title{
Erratum to: Wrinkling of a Stretched Thin Sheet
}

\author{
Eric Puntel · Luca Deseri · Eliot Fried
}

Published online: 9 April 2011

(C) Springer Science+Business Media B.V. 2011

\section{Erratum to: J Elast}

\section{DOI 10.1007/s10659-010-9290-5}

1. Equation (5) should read

$$
\mathbf{D}=\overline{\mathbf{E}}+\frac{1}{2} \overline{\mathbf{E}}^{2}+\frac{1}{2}(1-v \varepsilon) w,_{\alpha}\left(\mathbf{e}_{3} \otimes \mathbf{e}_{\alpha}+\mathbf{e}_{\alpha} \otimes \mathbf{e}_{3}\right)+\frac{1}{2} w,_{\alpha} w,_{\beta} \mathbf{e}_{\alpha} \otimes \mathbf{e}_{\beta}
$$

2. The axis labels in Fig. 4 are incorrect and those in Fig. 5 are ambiguous. They should be replaced as indicated in the figures on the second page of this erratum.

3. In the sentence "Consider now $\mathcal{C}_{1}^{*}$ and Figs. 1 and 2 b. For $n+1 \leq \kappa_{+}<n+2, n \in \mathbb{N}, \mathcal{C}_{1}^{+}$ belongs to the lower envelope of $\mathcal{C}_{1}^{+}$and $\mathcal{C}_{1}^{-}$." appearing two lines above equation (95), " $n \in \mathbb{N}$ " should be replaced by " $n$ even in $\mathbb{N}$."

4. Finally, the last sentence in the caption of Fig. 13 should read "The straight continuous line indicates the linear trend" instead of "The black line indicates the linear trend."

The online version of the original article can be found under doi:10.1007/s10659-010-9290-5.

\footnotetext{
E. Puntel

Dipartimento di Georisorse e Territorio, Università di Udine, via Cotonificio 114, 33100 Udine, Italy

L. Deseri

Dipartimento di Ingegneria Meccanica e Strutturale, Università di Trento, via Mesiano 77, 38023 Trento, Italy

E. Fried $(\bowtie)$

Department of Mechanical Engineering, McGill University, 817 Sherbrooke Street West, Montréal, Québec H3A 2K6, Canada

e-mail: eliot.fried@mcgill.ca
} 

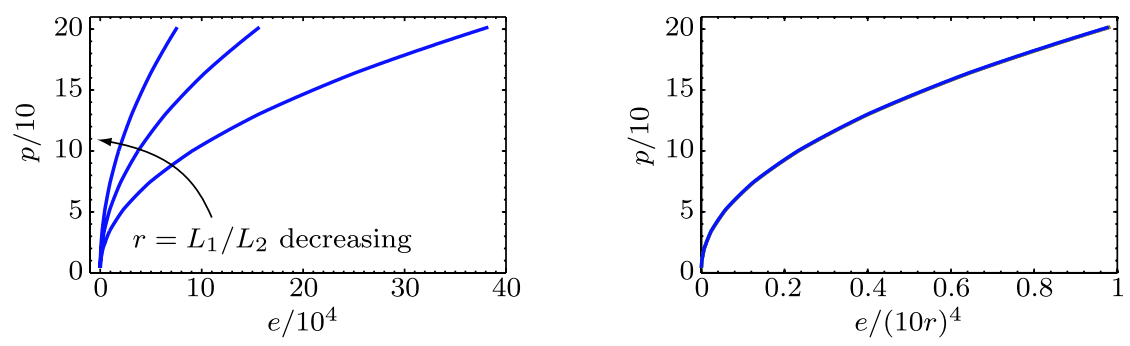

Fig. 4 (a) Plots of the minimum dimensionless effective compressive load $p$ versus $e$ for aspect ratios $r=L_{1} / L_{2}$ of $5 / 3,2$, and $5 / 2$. (b) The $p$ versus $e$ curves collapse to a single curve when $p$ is plotted versus $e / r^{4}$
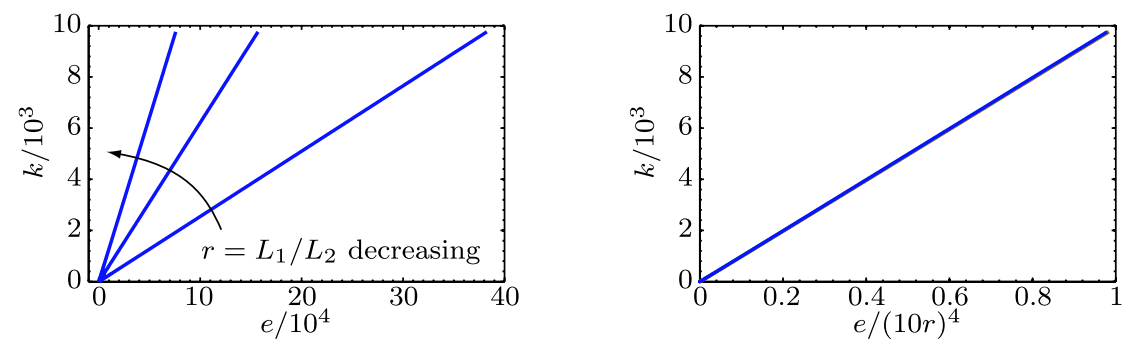

Fig. 5 (a) Plots of the dimensionless stiffness $k$ of the effective elastic foundation versus $e$ for aspect ratios $r=L_{1} / L_{2}$ of $5 / 3,2$, and $5 / 2$. (b) The $k$ versus $e$ curves collapse to a single curve when $k$ is plotted versus $e / r^{4}$ 